\title{
Zweidimensionale Bewegungsformen bei Vibrationsförderern
}

PROF. DR.-ING. KLAUS NENDEL

DIPL.-ING. THOMAS RISCH

\section{TECHNISCHE UNIVERSITÄT CHEMNITZ}

INSTITUT FÜR FÖRDERTECHNIK UND KUNSTSTOFFE

PROFESSUR FÖRDERTECHNIK

Vibrationsförderer stellen sowohl für Schüttgüter als auch für Stückgüter kleiner bis mittlerer Abmessungen einen wesentlichen Anteil der eingesetzten Zuführtechnik dar. Die dabei vom Fördergut erreichten Fördergeschwindigkeiten sind maßgeblich vom Betriebspunkt eines Vibrationsförderers abhängig und werden in der Praxis häufig durch die Betriebsfrequenz, die Schwingweite und den Wurfwinkel zur Horizontalen bestimmt. Der Einsatz von Schwingungsisolatoren zur Minderung der an die Umgebung übertragenen dynamischen Kräfte kann am Förderorgan Bewegungsformen verursachen, welche die Berechnung der resultierenden Fördergeschwindigkeit nach den bekannten Richtlinien nicht mehr ermöglicht. Anhand theoretischer und experimenteller Untersuchungen werden Analysemethoden zur Bestimmung zweidimensionaler Bewegungsformen vorgestellt und weiterführende Ansätze zur Simulation der Fördergutbewegung angeschnitten.

Vibratory conveyors represent an essential proportion of the feeding technology used for small and medium-sized bulk goods and piece goods. The obtained speed of the conveyed good is considerably dependent on the operating point of a vibratory conveyor and is often determined by the operating frequency, the amplitude and the throwing angle to the horizontal. The use of vibration isolators to reduce the dynamism transferred to the environment may cause movement patterns of the conveyor that make the calculation of the resulting conveying speed according to the known guidelines impossible. Analysis methods of two-dimensional movement patterns are introduced with the help of theoretical and experimental examinations. Further approaches to simulate movement patterns of conveyed goods are introduced.

\section{Einleitung}

Die Schwing- oder auch Vibrationsfördertechnik genannt, ist ein Spezialgebiet der Fördertechnik im Bereich der stetigen Förderprozesse. Die an die eingesetzte Technik gestellten Aufgaben, beziehen sich einerseits auf die Förderung innerhalb kleiner bis mittlerer Förderstrecken (meist Distanzen $<10 \mathrm{~m}$ ) und andererseits auf die Sortierung, Positionierung und Dosierung der Güter.

Im Bereich der Schüttgüter mit vorwiegend granularen Eigenschaften steht meist die Gutförderung im Vordergrund, die häufig innerhalb eines begleitenden Verarbeitungs- oder Veredelungsprozesses wie z.B. Klassierung oder Trocknung stattfindet. Im Umgang mit Stückgütern kleiner bis mittlerer Abmessungen, die sich innerhalb von Bunkereinheiten in einer ungeordneten Lage befinden, werden Schwingfördersysteme eingesetzt, um die Stückgüter in eine definierte Lage zu bringen, diese abzuführen und für anschließende Verarbeitungsprozesse zu positionieren. Zu typischen Produktvertreten gehören dabei beispielsweise Schrauben, Tabletten und kleine Baugruppen wie die Funktionskomponenten von Sprayflaschen. Der für diese Aufgaben notwendige materielle, energetische und technische Aufwand ist sehr gering im Vergleich zum Einsatz anderer Förder- und Handhabesysteme, wie z.B. sensorisch überwachten umlaufenden Zugmitteln oder gesteuerten Greiferelementen (Robotertechnik). Die zahlreichen Vorteile, wie z.B. der technisch einfache Aufbau, hohe Zuverlässigkeiten sowie das nahezu verschleißfreie Betriebsverhalten der Geräte im Dauereinsatz führten dazu, dass Vibrationsförderer bis heute einen Anteil von ca. 80\% der eingesetzten Zuführtechnik einnehmen. 


\section{Theoretische Grundlagen}

\subsection{Aufbau, Wirkungsweise und Klassifizierung}

Im Wesentlichen ist der Aufbau von Vibrationsförderern durch ein schwing- oder pendelfähig gelagertes Förderorgan gekennzeichnet, welches meist als Rinne (Förderrinne), Rohr oder Trog ausgebildet ist und mittels einer Erregerkraft $F_{\text {err }}(t)$ oder einer Wegvorgabe $s_{\text {err }}(t)$ in eine periodisch hin- und hergehende Bewegung versetzt wird. Diese Bewegung wird meist über die Frequenz, die Schwingweite (doppelter Maximalwert der Bewegungsamplitude) und den Anstellwinkel (Winkel zwischen der Horizontalen und der Bewegungsrichtung des Förderorgans) charakterisiert und als Betriebspunkt bezeichnet.
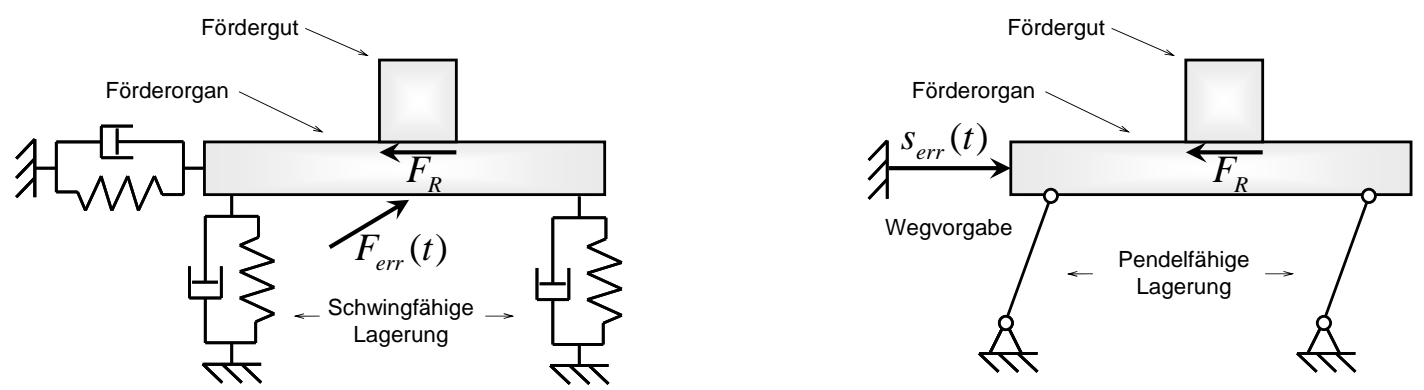

Abb. 1: Verallgemeinerter Aufbau eines Schwingförderers; Schwingsystem (links); Mechanismus (rechts)

Das Förderorgan beschleunigt das auf ihm liegende Gut bei seiner Bewegung in Förderrichtung über wirkende Reibungskräfte an den Kontaktflächen. Bei der Rückwärtsbewegung des Förderorgans behält das Fördergut aufgrund seiner Massenträgheit die ursprüngliche Bewegungsrichtung bei. Ist die Relativbewegung des Fördergutes zum Förderorgan innerhalb einer Bewegungsperiode in einer Richtung größer, so entsteht eine resultierende Fördergutbewegung, deren Betrag als Fördergeschwindigkeit bezeichnet wird.

Beim Mikrowurfprinzip zwingt die Bewegungsform des Förderorgans das Fördergut in eine Mikrowurfbewegung. Im einfachsten Falle bewegt sich das Förderorgan unter einem Wurfwinkel $\beta$ zur Horizontalen mit einer Betriebsfrequenz $f_{b}$ und einer Schwingweite $2 \hat{q}$. Aus dem Wurfwinkel $\beta$ resultiert folglich ein vertikaler Beschleunigungsanteil. Überschreitet der nach unten gerichtete vertikale Beschleunigungsanteil des Förderorgans die Erdbeschleunigung $\boldsymbol{g}$, so beginnt das Fördergut abzuheben und es kommt zum Wurf. Das Gut behält aufgrund seiner Massenträgheit die ursprüngliche Bewegungsrichtung bei und bewegt sich auf einer Mikrowurfparabel in Förderrichtung weiter. Fast alle Schwingförderer, die zur Förderung in der horizontalen Ebene oder unter einem geringen Steigungswinkel ausgelegt sind, arbeiten nach dem Mikrowurfprinzip.

Das Gleitprinzip wird durch den dauerhaften Kontakt zwischen Fördergut und Förderorgan charakterisiert. Folglich darf der nach unten gerichtete vertikale Beschleunigungsanteil bei der Bewegung des Förderorgans zu keinem Zeitpunkt die Erdbeschleunigung überschreiten. Eine Relativbewegung des Fördergutes ist dennoch möglich, wenn die Normalkraftkomponente des Gutes beim Hin- und Rückhub unterschiedliche Beträge aufweist. Bis auf wenige spezielle Ausnahmen, wird das Gleitprinzip bislang fast ausschließlich durch Schwingförderer realisiert, die als Schüttelrutschen ausgebildet sind.

\subsection{Berechnung der Fördergeschwindigkeit}

Um Schwingfördergeräte in Fertigungs- oder Verarbeitungsstrecken zu integrieren, ist es notwendig, deren Fördergutdurchsätze wie Volumen- oder Massenströme zu kennen. Die zunehmende Forderung nach logistisch effizient getakteten Teilstationen in Produktionsabläufen lässt oft nur sehr kleine Spielräume zu, von denen die Förderleistung abweichen darf. Anpassungen des Gutdurchsatzes können nur durchgeführt werden, wenn sich der Betriebspunkt bereits integrierter Geräte im Nachgang in erforderlichem Maße variieren lässt. Für die Dimensionierung und Erstauslegung ist es daher notwendig, dass dem Konstrukteur möglichst genaue Berechnungsvorschriften als Werkzeug zur Verfügung stehen, um die zu erwartende Fördergeschwindigkeit $v_{F}$ des Fördergutstromes berechnen zu können. 
In der Entwicklungsgeschichte der Schwingfördertechnik sind zahlreiche unterschiedliche Berechnungsmodelle vorgesellt worden, welche die Gutbewegung beim Fördervorgang in unterschiedlichster Detailtiefe beschreiben. In der Praxis hat sich jedoch die in der VDI 2333 enthaltene Berechnungsvorschrift zur Bestimmung der theoretischen Fördergeschwindigkeit durchgesetzt [VDI.65]. Diese Berechnungsvorschrift basiert auf den von Böttcher und Wehmeier entwickelten Modellen aus den sechziger Jahren und ist das Resultat einer analytischen Lösung des formulierten Problems.

$v_{t h}=\frac{g \cdot \bar{n}^{2}}{2 f_{B}} \cdot \cot \beta$

Die Vorteile dieser Berechnungsvorschrift liegen in der Einfachheit und der guten praktischen Tauglichkeit für Werte einer relativen Wurfzeit von $\bar{n}=0,8 \ldots 0,9$. Die Vereinfachungen und idealisierten Annahmen, die bei der Modellierung getroffen wurden, grenzen das Aussagespektrum dieser Berechnungsvorschrift jedoch nachhaltig ein. Die Vorschrift lässt sich nur unter Annahme einer harmonischen (sinusförmigen) Bewegung des Förderorgans anwenden. Es wird vorausgesetzt, dass das Gut nur innerhalb einer Periode springt und dass man ihm idealisierte Hafteigenschaften zuweisen kann. Impulseffekte und Relativbewegungen zum Förderorgan werden dabei vollständig ausgeschlossen. Haft- und Gleitreibungskräfte als Wechselwirkungen zwischen Fördergut und Förderorgan werden ebenfalls vernachlässigt. Des Weiteren ist es nach diesem Modell nicht möglich, Aussagen über den Zustand der Gleitförderung zu treffen, da die Berechnung der theoretischen Fördergeschwindigkeit stets an eine relative Wurfzeitkomponente gebunden ist, die sich beim Gleitförderprinzip zu Null ergibt.

All diese Einschränkungen zu Gunsten einer analytischen Lösung ermöglichen es in vielen Fällen nicht, die komplexen dynamischen Vorgänge beim Schwingfördervorgang umfassend zu beschreiben. Gerade dann, wenn die Bewegung des Förderorgans erheblich von einer harmonischen, linearen Bahn abweicht, was vor allem bei kleineren Schwingförderern (z.B. Zuführ- und Montagetechnik) verstärkt auftreten kann, kommt es zu enormen Abweichungen der berechneten mit den tatsächlich beobachteten Fördergeschwindigkeiten, bis hin zur Umkehr der beabsichtigten Förderrichtung.

\section{2D-Bewegungsformen bei Vibrationsförderern}

\subsection{Ursachen von 2D-Bewegungsformen}

Es gibt unterschiedliche Ursachen für die Entstehung zweidimensionaler Schwingformen bei Vibrationsförderern. Sie können sowohl beabsichtigt als auch unbeabsichtigt generiert werden. Jedoch gerade bei kleineren Schwingfördergeräten aus dem Bereich der Zuführ- und Montagetechnik werden die dadurch hervorgerufenen Auswirkungen auf den Fördervorgang ersichtlich.

Die von Vibrationsförderern an die Umgebung übertragenen Kräfte müssen in vielen Fällen minimiert werden, um periphere Anlagen in ihrer Funktionsweise nicht zu beeinflussen. Dazu werden die Prinzipien des Massenund Drehmomentenausgleichs sowie das Prinzip der Schwingungsisolation angewandt. Die Schwingungsisolatoren stellen jedoch in ihrer Funktion zusätzliche Federelemente dar, die die dynamischen Eigenschaften des gesamten Feder-Masse-Systems Schwingförderer nachhaltig beeinflussen.
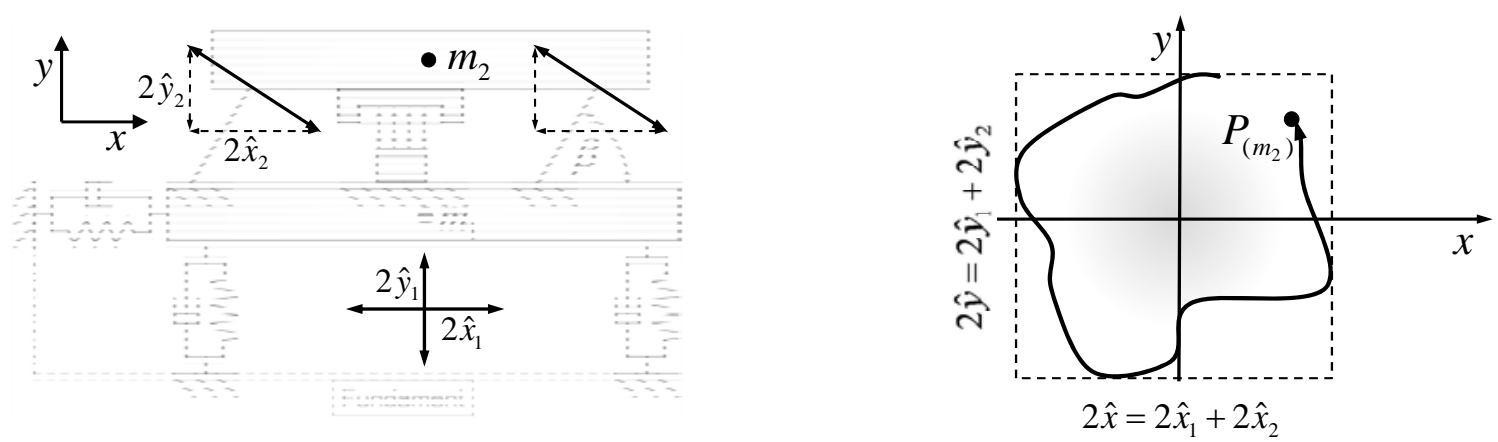

Abb. 2: Freiheitsgrade eines schwingungsisolierten Vibrationsförderers, Förderorgan ist auf Blattfedern gelagert (links); beliebige zweidimensionale Bewegungsform (Bahnkurve) des Förderorgans (rechts)

Zusätzlich zur Bewegungsfreiheit in x-Richtung, besitzen die Teilmassen vertikale Freiheitsgrade. Diese resultieren aus der Federlagerung des Gestellrahmens $m_{1}$ in y-Richtung. In der Praxis wird dieser Freiheitsgrad 
der Bewegung meist vernachlässigt, da die Schwingungsamplituden des Gestellrahmens in vertikaler Richtung meist wesentlich geringer ausgeprägt sind als die Amplituden in Förderrichtung. Aber gerade dieser vertikale Freiheitsgrad, ist häufig die Ursache für das Entstehen zweidimensionaler Bewegungsformen am Förderorgan und kann den Fördervorgang enorm beeinflussen.

Die Position des Förderorgans $P_{\left(m_{2}\right)}$ kann zu jedem Zeitpunkt $t$ mit zwei Koordinaten $x(t)$ und $y(t)$ beschrieben werden. Die Spur von $P_{\left(m_{2}\right)}$ wird als Bahnkurve oder Bewegungsform bezeichnet. In Abb. 2 ist eine beliebige zweidimensionale Bewegungsform dargestellt. Prinzipiell kann die Bahnkurve jeden möglichen Verlauf annehmen, muss aber immer geschlossen sein, da es sich beim Schwingförderprozess um einen periodischen Vorgang handelt. Sie entspricht der Schwingungsantwort des Gesamtsystems und lässt sich nach dem Superpositionsprinzip als Überlagerung der Teilschwingungskomponenten in der jeweiligen Koordinatenrichtung darstellen.

\subsection{Beschreibung harmonischer Bewegungsformen}

Aus der Periodizität des Schwingfördervorganges folgt, dass die Absolutkoordinaten Wertepaare bilden, die sich mit einer bestimmten Frequenz zeitlich wiederholen. D. h., es bildet sich als Überlagerung beider Bewegungskomponenten in jedem Fall eine geschlossene Bahnkurve $y(x)$ heraus. Bewegt sich das Förderorgan sowohl in vertikaler als auch in horizontaler Richtung sinusförmig (harmonisch), entsteht bei der Überlagerung eine harmonische Bewegungsform 1. Ordnung. Erreichen beide Bewegungskomponenten ihr Auslenkungsmaximum bzw. ihr Auslenkungsminimum nicht zeitgleich, spricht man von einer Phasenverschiebung der Teilschwingungskomponenten, die mit $\varphi$ bezeichnet wird.
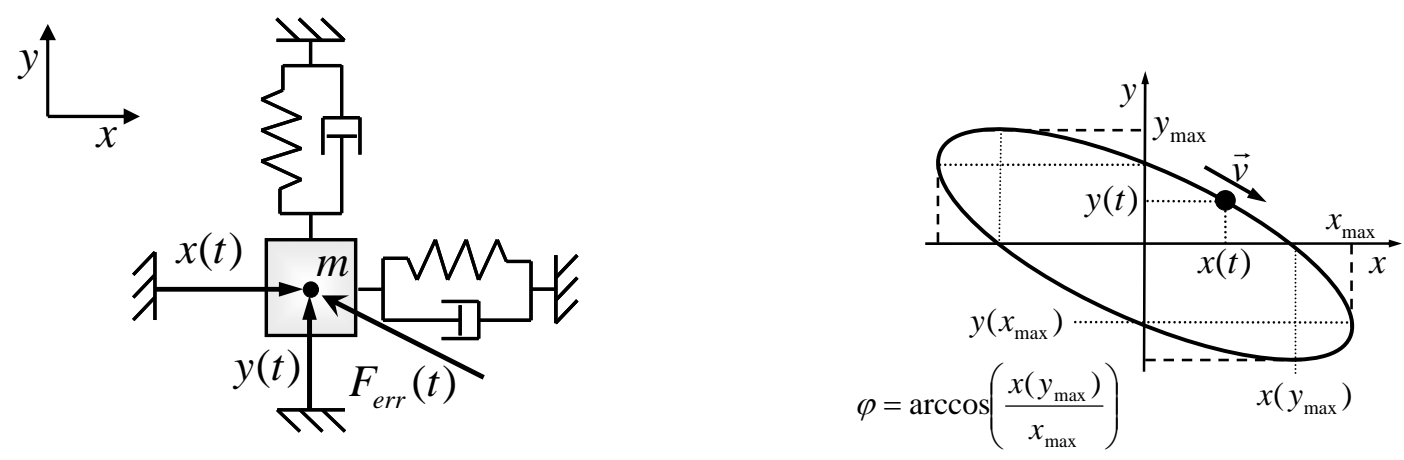

Abb. 3: Harmonische Bewegungsform 1. Ordnung als Resultat der Superposition einer vertikalen und einer horizontalen Teilschwingungskomponente; eine Phasenverschiebung führt zu einer elliptischen Bewegungsform

Harmonische Bewegungsformen 1. Ordnung lassen sich technisch relativ einfach erzeugen, da sie der Schwingungsantwort linearer Feder-Masse-Systeme entsprechen. Bleibt die Dämpfung der FederDämpferelemente bei der Modellierung vernachlässigt, können lediglich Phasenverschiebungen von $\varphi=0$ oder $\varphi=\pi$ auftreten. Die Masse aus Abb. 3 schwingt in jedem Fall mit der Frequenz der Erregung. Je nach dem, ob die skizzierten Federelemente über- oder unterkritisch zur Masse $m$ abgestimmt sind, ist die Schwingungsantwort entweder genau gleichphasig oder genau gegenphasig zur Erregung.

Wird die Dämpfung nicht vernachlässigt, ist jede Phasenverschiebung zwischen $0<\varphi<2 \pi$ möglich und kann durch geeignete Wahl der Steifigkeit des horizontalen und des vertikalen Federelementes generiert werden.

Durch den Einsatz von herkömmlichen Blatt- oder Gummifederelementen, die horizontal und vertikal anzuordnen sind, und einfacher Antriebseinheiten, welche die Teilschwingungen in beide Richtungen anregen, lassen sich zweidimensionale Bewegungsformen gezielt generieren. Bei vielen Schwingförderern treten solche Bewegungsformen oft unbeabsichtigt auf, bleiben meist unerkannt oder werden bei der Berechnung der Fördergeschwindigkeit einfach vernachlässigt. Dies führt nicht selten zu enormen Abweichungen der Berechnungsergebnisse der zu erwartenden Fördergeschwindigkeit. 
Zweidimensionale Bewegungsformen können sehr komplexe Formen annehmen, die mittels Überlagerung zweier harmonischer Funktionen nicht mehr ausreichend beschrieben werden können. Dennoch lässt sich jedes periodische Signal als unendliche Reihe harmonischer, bzw. trigonometrischer Funktionen darstellen. Diese Eigenschaft wird genutzt, um eine verallgemeinerte Möglichkeit der Beschreibung von Bewegungsformen $k$-ter Ordnung zu formulieren.

$$
\begin{aligned}
& x(t)=\sum_{n=0}^{k} C_{x n} \cdot \cos \left(n \Omega t+\varphi_{x n}\right) \\
& y(t)=\sum_{n=0}^{k} C_{y n} \cdot \cos \left(n \Omega t+\varphi_{y n}\right)
\end{aligned}
$$

Die Interpretation von Bewegungsformen als trigonometrische Reihen weisen für weiterführende Untersuchungen zahlreiche Vorteile auf. Es lässt sich nahezu jede denkbare Bahnkurve konstruieren, wobei schon mit einer geringen Anzahl von Reihenglieder $(k \leq 5)$ sehr gute Annäherungen an experimentell aufgenommene Schwingbilder erzielt werden können. Experimentelle Daten lassen sich somit in Form einer Koeffizientenmatrix nachbilden und in einer einfachen Datenbank dokumentieren. Des Weiteren weisen trigonometrische Reihen die Eigenschaften der Stetigkeit und Differenzierbarkeit auf, was zusätzlich die Eignung der Reihen als Inputfunktionen für ein numerisches Simulationsprogramm bestätigt.

$$
\stackrel{(n)}{x(t)}=\frac{d^{n} x}{d t^{n}}=\sum_{n=0}^{k} C_{x n} \cdot n \Omega^{n} \cdot \frac{d^{n}\left(\cos \left(n \Omega t+\varphi_{x n}\right)\right)}{d t^{n}}
$$

\section{Experimentelle Untersuchungen}

Um die Auswirkungen zweidimensionaler Bewegungsformen auf den Schwingfördervorgang experimentell zu untersuchen, wurde folgender Versuchsaufbau konzipiert. Unterschiedliche Vibrationsförderer, bei denen zweidimensionale Bewegungsformen am Förderorgan entstehen, wurden mittels Lasertriangulationssensoren im Betriebszustand vermessen. Dabei wurden zeitgleich die horizontalen und vertikalen Weg-Zeit-Verläufe sowie das Bewegungsverhalten des Fördergutes aufgezeichnet. Durch die gemeinsame zeitliche Basis der Messignale ist es möglich, die Reaktion des Fördergutes auf eine bestimmt Bewegungsform auszuwerten.

Die Vertikalbewegung des Förderorgans wurde an zwei Punkten (links- und rechtsseitig) aufgezeichnet, um die Stabilität der Bewegungsform über die gesamte Länge des Förderorgans einschätzen zu können. Durch diese Methodik lassen sich Taumel- oder Kippbewegungen des Förderorgans detektieren und Rückschlüsse auf geeignete Messstellen ziehen. Zusätzlich zur messtechnischen Untersuchung mittels Lasertriangulation wurden High-Speed-Aufnahmen der Fördergutbewegung angefertigt, die einerseits zur visuellen Unterstützung der Sensorsignale und andererseits als Modellierungshilfe geeigneter Algorithmen zur Beschreibung des Förderprozesses dienen sollen.
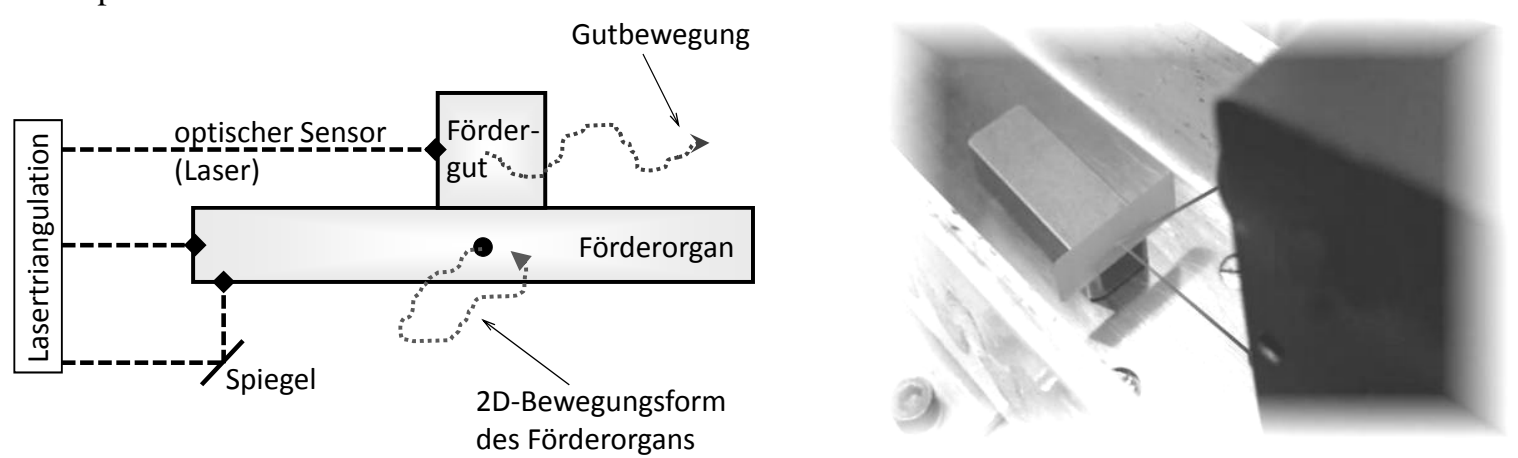

Abb. 4: Versuchsaufbau zur Bestimmung der Bewegungsform und der resultierenden Fördergutbewegung mittels Lasertriangulation (links); Aufzeichnung der Reaktion des Fördergutes am Versuchsstand (rechts) 
Der durchschnittliche Anstieg des Verlaufs $p(t)$ aus Abb. 5 entspricht der mittleren Fördergeschwindigkeit des Gutes als Reaktion auf die 2D-Bewegungsform des Förderorgans. Den ausgewählten Messwerten kann eine Betriebsfrequenz von $50 \mathrm{~Hz}$ und eine Schwingweite von 0,8 mm bei einer resultierenden mittlern Fördergeschwindigkeit von ca. 2,7 m/min zugeordnet werden.
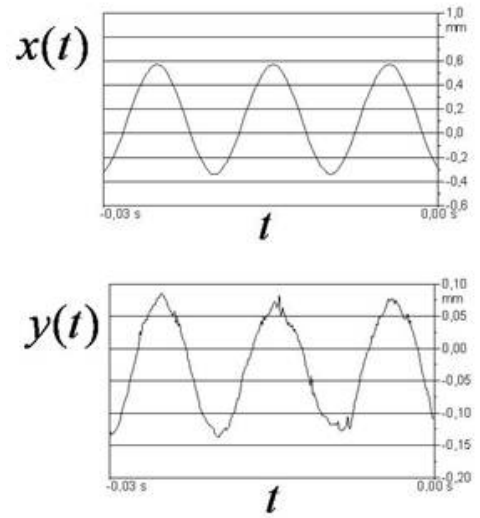

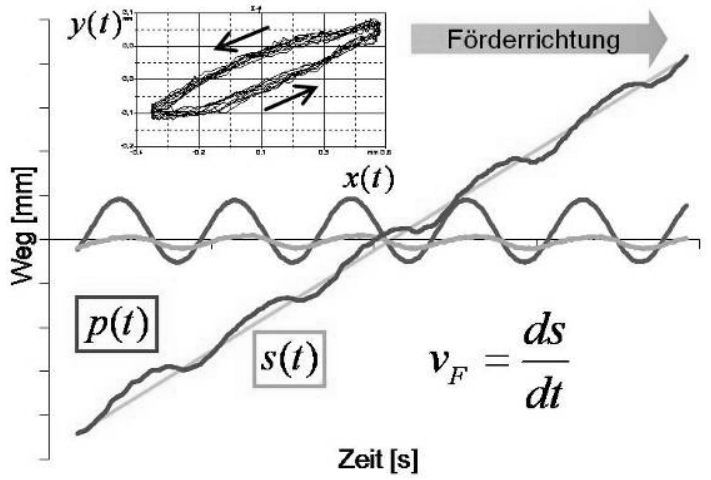

Zeit [s]

Abb. 5: Beispielmessung: ausgewählte Sensorsignale vom Förderorgan (links); Überlagerung der Sensorsignale auf zeitlich gleicher Basis, Kennzeichnung relevanter Größen (rechts)

Die aufgezeichneten Sensordaten wurden mittels diskreter Fouriertransformation über eigens entwickelte Makroprogramme ausgewertet und in Form einer Koeffizientenmatrix dokumentiert. Diese Datenstruktur bildet den Ausgangspunkt der Verifizierung eines noch in der Entwicklung befindlichen mathematischen Modells zur zeitdiskreten Berechnung des Fördergutverhaltens bei zweidimensionalen Bewegungsformen. Es wurden bereits Einzelverifikationen mit dem Ergebnis durchgeführt, dass die berechneten mittleren Fördergeschwindigkeiten im Durchschnitt um weniger als 10 \% von den experimentell ermittelten Daten abweichen.

\section{Zusammenfassung und Ausblick}

An der Professur für Fördertechnik der Fakultät für Maschinenbau der TU Chemnitz wurden unterschiedliche Vibrationsförderer experimentell untersucht, bei denen 2D-Bewegungsformen des Förderorgans im Betriebspunkt auftreten. Dazu wurden gleichzeitig die Bewegungsform des Förderorgans und die daraus resultierende Bewegung des Fördergutes berührungslos mittels Lasertriangulationssensoren aufgezeichnet. Die aus den diskreten Sensorsignalen erhaltenen Weg-Zeit-Verläufe werden mittels diskreter FOURIER-Analyse ausgewertet und in Form eine FOURIER-Koeffizientenmatrix aufgearbeitet und in einer Datenbank hinterlegt. Diese experimentellen Daten dienen dabei sowohl der empirischen Auswertung als auch der Verifizierung und Erweiterung eines eigens entwickelten mathematischen Modells, das sich derzeit auf das Anwendungsfeld von Stückgütern konzentriert. Auf Basis dieses mathematischen Modells soll schließlich ein Simulationswerkzeug entwickelt werden, mit dessen Hilfe Fördergeschwindigkeiten wesentlich präziser und detaillierter berechnet werden können, als dies mit den bereits bekannten Berechnungsvorschriften nach der VDI-Richtlinie möglich ist. Es lassen sich die Fördergutreaktionen auf beliebige Bewegungsformen des Förderorgans zeitdiskret vorhersagen und damit umfangreiche theoretische Untersuchungen hinsichtlich der relevanten Bewegungsparameter durchführen. Daraus lassen sich optimale Bewegungsgesetze für Vibrationsförderer ableiten, die schließlich eine gezielte Entwicklung und konstruktive Umsetzung effizienter Geräte ermöglichen. 


\section{Literatur}

[Trö.06]

Trölß, J.: Komplexe Zahlen und Funktionen, Vektoralgebra und analytische Geometrie, Matrizenrechnung, Vektoranalysis. Wien, New York: Springer-Verlag, 2006. ISBN 3-211-29687-5.

[Opp.04]

Oppenheim, A. V.; Schafer,R. W.: Zeitdiskrete Signalverarbeitung. 2. überarbeitete Auflage [Neuübers.]. München, u. a.: Pearson Studium, 2004. ISBN 3-8273-7077-9.

[Bri.95]

[Ben.05]

[VDI.65]

Brigham, E. O.: Schnelle Fourier-Transformation / übers. Von Seyed Ali Azizi. 6., korrigierte Auflage. München, Wien: Oldenbourg Verlag, 1995. ISBN 3-486-231774.

[Böt.57]

[Weh.61]

[Hab.84]

Benker, H.: Differentialgleichungen mit Mathcad und Matlab. 1. Auflage. Berlin, Heidelberg [u.a.]: Springer Verlag, 2005. ISBN 3-540-23440-3.

VDI-Richtlinie 2333. Schwingförderer für Schüttgut. Ausg. 10. 1965.

Böttcher, S.: Beitrag zur Klärung der Gutbewegung auf Schwingrinnen. Hannover: Diss. Technische Hochschule Hannover, 1957.

Wehmeier, K.-H.: Untersuchungen zum Fördervorgang auf Schwingrinnen. Hannover: Diss. Technische Hochschule Hannover, 1961.

Habenicht, D.: Grundlagenuntersuchungen zur Werkstückgleitförderung in Schwingzuführsystemen. Hannover: Diss. TU Hannover, 1984.

[Der.06]

Dresig, H.; Holzweißig, F.: Maschinendynamik. 7., bearbeitete Auflage. Berlin, Heidelberg: Springer-Verlag, 2006. 